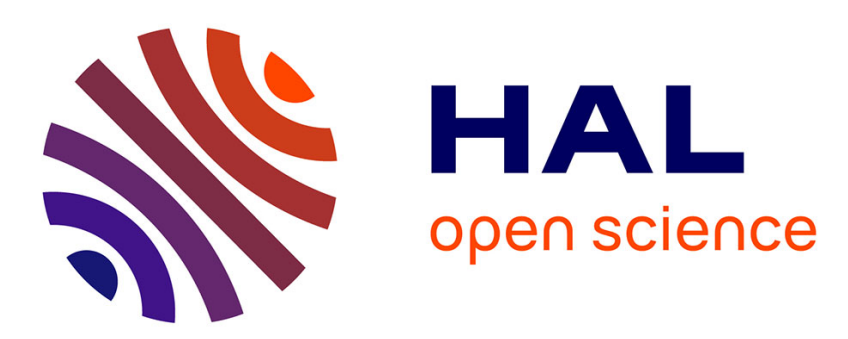

\title{
Ultrarare marine microbes contribute to key sulphur-related ecosystem functions
}

\author{
Dimitri Kalenitchenko, Nadine Le Bris, Erwan Peru, Pierre Galand
}

\section{To cite this version:}

Dimitri Kalenitchenko, Nadine Le Bris, Erwan Peru, Pierre Galand. Ultrarare marine microbes contribute to key sulphur-related ecosystem functions. Molecular Ecology, 2018, 27 (6), pp.1494-1504. 10.1111/mec.14513. hal-02342179

\section{HAL Id: hal-02342179 https://hal.sorbonne-universite.fr/hal-02342179}

Submitted on 31 Oct 2019

HAL is a multi-disciplinary open access archive for the deposit and dissemination of scientific research documents, whether they are published or not. The documents may come from teaching and research institutions in France or abroad, or from public or private research centers.
L'archive ouverte pluridisciplinaire HAL, est destinée au dépôt et à la diffusion de documents scientifiques de niveau recherche, publiés ou non, émanant des établissements d'enseignement et de recherche français ou étrangers, des laboratoires publics ou privés. 
1 Ultra-rare marine microbes contribute to key sulfur related ecosystem functions

2

3 Dimitri Kalenitchenko ${ }^{\mathrm{a}}$, Nadine Le Bris ${ }^{\mathrm{a}}$, Erwan Peru ${ }^{\mathrm{a}}$, Pierre E. Galand ${ }^{\mathrm{a} 1}$

4

5 a Sorbonne Universités, UPMC Univ Paris 06, CNRS, Laboratoire d'Ecogéochimie des

6 Environnements Benthiques (LECOB), Observatoire Océanologique, Banyuls sur Mer,

7 France

8

9

10

11

12

13

14

15

16

17 Keywords: rare biosphere, bacteria, functional redundancy, sulfide, diversity, colonization,

18 succession, sulfur cycle

19

20

21

22

23

24

$25{ }^{1}$ To whom correspondence should be addressed. E-mail: pierre.galand@obs-banyuls.fr. 
The description of a rare biosphere within microbial communities has created great interest because microbes play a fundamental role in the functioning of all ecosystems on earth. Despite recent progress in understanding the ecology of the rare biosphere, the concept itself is still discussed and fundamental questions remain. Here, we target the seed bank compartment of the rare biosphere, assess the level of rarity at which microorganisms are still able to colonize an ecosystem and investigate whether rare species are functionally redundant. Using an original experimental design where wood in aquaria was inoculated with increasingly diluted coastal seawater, we show that bacteria that represented as few as $0.00000002 \%$ of the cells in the environment (or 1 cell in $10 \mathrm{~L}$ of seawater) were still able to grow and play key roles within the ecosystem. Our experiment further showed that some bacteria can be replaced by others that have the potential to fulfill the same metabolic tasks. This finding suggests some functional redundancy within bacterial species. However, when ultra-rare bacteria were progressively removed, productivity was reduced, and below a certain threshold some processes were lost, and the function of the ecosystem was altered. Overall the study shows that bacteria that are not detected by high-throughput sequencing approaches are nevertheless viable and able to colonize new ecosystems, suggesting the need to consider ultra-rare microbes in the marine environment. 
Microbial communities play a fundamental role in the functioning of all ecosystems on earth (Falkowski et al. 2008; McFall-Ngai et al. 2013). In the sea, they mediate key biogeochemical cycles and are involved in nutrient cycling, primary production, organic matter mineralization and ultimately climate regulation (Kirchman 2010). These processes are often conducted by a few dominant and active species that are present in the environment at a given time (PedrósAlió 2006). Such a feature is reflected by the representation of the distribution of microorganisms along a skewed rank-abundance curve that shows the presence within a community of a few dominant species and many less abundant organisms (Curtis et al. 2002; Pedrós-Alió 2012). This long tail of rare microbes contains most of the tremendous diversity of a microbial community (Crespo et al. 2016).

This large number of rare species was coined the "rare biosphere" following the first application of high-throughput sequencing to describe marine microbial communities (Sogin et al. 2006). The rare marine biosphere was first hypothesized to reflect the stochastic and potentially unlimited dispersal capacity of microbes in the ocean. Traces of these dispersed microorganisms would eventually be found everywhere. Furthermore, following Baas Becking's statement that "everything is everywhere, but the environment selects", these microbes would represent a seed bank containing organisms that can grow when the conditions become favorable (Jones \& Lennon 2010; Lennon \& Jones 2011; Pedrós-Alió 2006). However, it was shown that the rare biosphere represented a more complex picture. It has patterns of biogeography that suggests the occurrence of selection processes (Galand et al. 2009). Furthermore, some rare microbes are active and at times are more active than the abundant members of the community (Campbell et al. 2011; Jones \& Lennon 2010), which indicates that the rare biosphere is not only composed of a seed bank of dormant microorganisms. It thus appears that there are large ecological differences between different 
compartments of the rare biosphere (Alonso - Sáez et al. 2015; Hugoni et al. 2013; PedrósAlió 2012). Despite recent progress in understanding the ecology of the rare biosphere, the concept itself is still discussed, and fundamental questions remain. For instance, some data suggest that some of the observed microbial diversity results from methodological artifacts (Kunin et al. 2010; Lee et al. 2012; Patin et al. 2013) or that the observed diversity does not represents viable microorganism but instead reflects free or dead DNA (Carini et al. 2016; Lynch \& Neufeld 2015). In addition, although sequencing now allows the in-depth probing of the richness of the rare biosphere (Crespo et al. 2016), it was recently debated that there may not be as many rare microbes as originally thought (Amann \& Rossello-Mora 2016; Amann \& Rosselló-Móra 2016; Lennon \& Locey 2016). However, there have been few if any convincing experiments designed to explore the limits of rarity in natural samples. The existence of the rare biosphere also begs the question of why there are so many microbial species in the sea. High levels of diversity have been early explained by the presence in the environment of different taxa that are able to conduct the same metabolic processes. The paradigm of functional redundancy has long been assumed in microbial ecology (Bell et al. 2005; Finlay et al. 1997), and the assumption is that a change in microbial community composition will not have consequences for microbial-mediated processes (Allison \& Martiny 2008). The recent use of metagenomic profiles revealed high functional redundancy for marine microbes at global scale (Sunagawa et al. 2015). Inversely, the fact that some bacterial communities that are distinct in composition show different functions (Leff et al. 2015) indicates that the paradigm of redundancy does not always hold in natural microbial communities, as shown recently in soil ecosystems (Fierer et al. 2013). In aquatic environments, recent findings showing intrapopulation complementary (Garcia et al. 2015) support the theory of a high level of functional complementary in marine microorganisms 
100 illustrated by reductive genomic evolution or genome streamlining (Giovannoni et al. 2014;

101 Morris et al. 2012).

Here, we focus on the seed bank compartment of the rare biosphere, which was

103 conceptualized by Pedrós-Alió $(2006,2012)$ as rare taxa that are not growing but that become

104 abundant when conditions become favorable, and investigate the extent of its diversity and

105 viability. More precisely, the goal of this study was to determine a threshold of abundance at

106 which rare organisms can still successfully colonize a complex substrate, to test whether the

107 rare species are functionally redundant and to verify whether they can be detected in the

108 environment. We earlier showed that wood incubated in seawater is a useful tool for testing

109 hypothesis about the functional ecology of communities (Kalenitchenko et al. 2016). Here,

110 wood incubated in aquaria were inoculated with different dilutions of coastal seawater, and

111 the growth of the bacterial communities was followed both within the wood and on the wood

112 surface, with a special focus on the bacteria involved in the sulfur cycle. These keystone

113 species are responsible for key ecosystem processes in the ocean. They are active during the

114 degradation of large organic falls on the sea floor (Bienhold et al. 2013; Kalenitchenko et al.

115 2015; Kalenitchenko et al. 2017), the mineralization of organic matter in marine sediments

116 (Jørgensen 1982) and biomass production at hydrothermal vents or cold seeps (Campbell et al.

117 2006; Martin et al. 2008). We chose to target these organisms because of their global

118 importance and because their activity can be conveniently monitored.

120 Materials and methods

121 Experimental set up

122 Wood logs originating from a same pine tree (Pinus pinea) in the Banyuls sur Mer

123 ' Biodiversarium' botanical garden were cut the day before the experiment started. The

124 experimental design consisted of four separate $40-\mathrm{L}$ aquaria that each contained four $15 \mathrm{~cm}$ in 
125 diameter and $10 \mathrm{~cm}$ in length, fresh (non sterile), wood logs (Kalenitchenko et al. 2016). We

126 did not want to take the risk of changing the integrity of the chemical composition of the

127 wood by autoclave (possible impact on sugars). The aquaria were initially filled with

128 increasing dilutions of coastal seawater from the SOLA station in Banyuls Bay sampled at 3-

129 m depth on 27 January 2015. Seawater filtered through a UF100LL filtration module

130 (Polymem, Castanet-Tolosan, France) with a $0.01 \mu \mathrm{m}$ pore size membrane was used for the

131 dilutions. The first aquaria was started with $100 \%$ (1/1 treatment) off coastal unfiltered

132 seawater, the second with $4 \mathrm{~L}$ of coastal water and $36 \mathrm{~L}$ of filtered water (1/10 treatment), the

133 third with $0.4 \mathrm{~L}$ of coastal water and $39.6 \mathrm{~L}$ of filtered water $(1 / 100$ treatment $)$ and the last

134 with $100 \%$ filtered water (filtered treatment). Wood logs were immersed without water

135 renewal for the first 7 days of the experiment after which, the aquaria water was renewed with

136 piped in coastal seawater filtered through the $0.01 \mu \mathrm{m}$ pore size filtration module (Polymem)

137 at a flow rate that allowed at least 2 renewals per day. The experiment lasted for 28 days.

138 One log was removed and sampled from each aquarium after 7 days (7d), 14 days

139 (14d), 21 days (21d) and 28 days (28d). At each sampling time, four replicate 1-cm long cores

140 were extracted from each log using a 4.35-mm-wide increment core borer. Mats growing on

141 the wood surface were sampled when present (21d and 28d) by scratching the entire wood

142 surface with a scalpel blade.

\section{DNA extraction and sequencing}

145 The microbial mat samples were lysed and wood core samples were powdered as described

146 earlier (Kalenitchenko et al. 2016; Kalenitchenko et al. 2015). The cell lysate was then

147 transferred to the Maxwell ${ }^{\circledR} 16$ Blood DNA Purification kit cartridge and processed

148 automatically with the Maxwell ${ }^{\circledR} 16$ automated extractor (Promega, Fitchburg, MA, USA). 
DNA was also extracted from the original coastal seawater as described previously (Galand $e t$

150 al. 2015). See the supplementary material and methods for a full description of the procedure.

A portion of the $16 \mathrm{~S}$ rRNA gene was amplified using bacteria specific primers, $28 \mathrm{~F}$ SRP099419 and BioProject accession no. PRJNA374511).

\section{Sequence analysis}

159 The 16S rRNA sequences were processed by following (Galand et al. 2015). In brief,

160 sequences were paired, producing ca. $500 \mathrm{bp}$ fragments, quality trimmed, and chimeras were

161 removed. Sequences were grouped in operational taxonomic units (OTU) at 97\% similarity

162 using the Uclust algorithm (Edgar 2010). The taxonomy of the most abundant sequence of

163 each OTU was assigned using the SILVA SSU 123 database (Pruesse et al. 2007). All

164 samples were resampled down to 5094 sequences per sample.

165 To identify the possible presence of rare species in seawater we used a dataset of sequences

166 from the Banyuls Bay microbial observatory time series. Samples have been gathered twice a

167 month from 2007 to 2014. DNA has been extracted and samples sequenced as described

168 earlier (Galand et al. 2015). A total of 7,418,629 sequences were used to search for rare

169 species in the time series. The occurrence of sequences that were not detected in the time

170 series was calculated to be $<1 / 7,418,629$, which is $<0.00001 \%$.

171 Bacterial diversity was estimated by calculating the Shannon diversity index (H') and the 172 standardized effect size (SES) (Kembel et al. 2010), which is a standardized measure of 173 phylogenetic diversity. Phylogenetic diversity was computed using the Picante package 174 (Kembel et al. 2010) in R. To calculate SES, representative sequences from each OTU were 
175 aligned using MUSCLE (Edgar 2004), and the alignment was then cleaned to remove nonoverlapping sequence regions. A phylogenetic tree was constructed using FASTTREE (Price et al. 2010). The observed phylogenetic diversity was compared to the average phylogenetic diversity in a randomly generated community (null model) and divided by the standard deviation of the phylogenetic distances in the null model (Webb et al. 2008). The null model randomizes community data matrixes with the independent swap algorithm to maintain the species occurrence frequency and sample species richness (Kembel 2009). Positive SES values indicate greater phylogenetic distance among co-occurring species than expected by chance, whereas negative values indicate small phylogenetic distance.

The Bray Curtis similarity index was computed to compare the community composition between samples with an MDS analysis. The effect of dilution and time factors on composition differences was tested with PERMANOVA in the program PAST (Hammer et al. 2001). To identify OTUs or bacterial orders that had a significant difference in abundance between groups, we used a nested ANOVA with the tools described in (McDonald 2014). Differences in diversity (Shannon) were tested with ANOVA. Two outlier samples (1/1: 14dReplicate1, 1/10/ 21d-Replicate1) were removed from the analysis.

\section{Flow cytometry and most probable number}

Heterotrophic prokaryotic abundance in the aquaria water was measured by flow cytometry (Supplementary methods). We used a method that is commonly used in microbiology, called the most probable number method (MPN) (Oblinger \& Koburger 1975), to estimate the number of cells belonging to the OTUs that we monitored. This method relies on dilutions and the observation of microbial growth to estimate the number of cells present in a starting solution. To do so, we counted the number of replicate wood samples in which we detected the OTUs of interest at the end of the experiment (28d). We then used an MPN table to 
estimate the number of cells in the original seawater sampled from Banyuls Bay. Their

201 frequency was calculated by dividing the estimated number of cells by the total number of cell counted by flow cytometry.

\section{Sulfide detection}

205 Continuous monitoring of sulfide was performed using autonomous potentiometric sensors

206 (NKE, Hennebont, France) equipped with lab made $\mathrm{Ag} / \mathrm{Ag}_{2} \mathrm{~S}$ electrodes (Le Bris et al. 2012)

207 to detect when sulfide started to diffuse at the surface of the wood logs. To measure the

208 sulfide through the microbial mats, microsensor profiling was performed using amperometric

209 microsensors (H2S100) and a picoammeter (PA2000) (UNISENSE, Aarhus, Denmark).

210 Sulfide concentrations were profiled vertically, perpendicular to the surface of the wood, with

211 a resolution of $250 \mu \mathrm{m}$ at each sampling time using a micromanipulator. Calibrations were

212 performed by standard additions of $\mathrm{Na}_{2} \mathrm{~S}$ stock solution in seawater as described in Laurent et

213 al. (2009) (Laurent et al. 2009) for the potentiometric electrodes. The amperometric electrode

214 was calibrated at $\mathrm{pH}$ of 6, 6.5, 7 and 7.5 using a Titrando $\mathrm{pH}$ controller (Metrohm, Herisau,

215 Switzerland). The slope of the electrode response to $\mathrm{H}_{2} \mathrm{~S}$ was thus confirmed to be $\mathrm{pH}$

216 independent and was calculated combining the whole calibration series. The total free sulfide

217 concentration (i.e. both $\mathrm{H}_{2} \mathrm{~S}$ and $\mathrm{HS}$ ) is determined from the $\mathrm{pH}$ variability at the wood-water

218 interface. To calculate the $\mathrm{HS}^{-}$fraction, we used a $\mathrm{pKa}_{1} *$ of 6.7 at $13^{\circ} \mathrm{C}$ and salinity 37

219 (Rickard \& Luther 2007).

The $\mathrm{pH}$ at the wood surface and wood water interface was measured with $1 \mathrm{~mm}$

221 resolution with a mini electrode (1.5 mm diameter) (Diamond General Co., Ann Arbor, USA)

222 coupled with the sulfide microsensor. To prevent sulfide profiles from being disturbed by the

223 larger $\mathrm{pH}$ electrode, the tips of both electrodes were $1 \mathrm{~cm}$ apart. Triplicates of the combined 
$224 \mathrm{pH}$ and $\mathrm{H}_{2} \mathrm{~S}$ profiles were acquired to determine mean concentration of total sulfide and the

225 corresponding standard deviation, which reflects the heterogeneity of the mat.

\section{Results}

\section{Sulfide concentrations}

229 We continuously measured sulfide concentrations inside the wood during the course of the 230 experiment. In the undiluted treatment, sulfide was first detected inside the wood after 13 231 days of incubation. In the 1/10 dilution, sulfide was detected after 21 days, and in the 1/100 232 dilution, the electrode potential only showed a small shift from baseline $(<7 \mathrm{mV})$, which 233 indicated that no significant amount of sulfide built up at the wood surface. In the aquaria 234 with filtered water only, the sulfide electrode potential in the wood did not change over the 235 experiment (Fig. 1a).

236 Surface mat sulfide profiles obtained with amperometric microsensors also reveal 237 marked differences between the treatments (Fig. 1b). The mats were first visible at 21d, and 238 the maximum sulfide concentration was higher in the undiluted experimental mats than in the 239 1/10 dilution mats. No mats were visible at the other dilutions. At 28d, the undiluted mat had 240 thicker sulfide layer and higher maximum sulfide concentrations at the wood surface 241 compared to those in the 1/10 dilution treatment (Fig. 1b). No sulfide was detected on the $242 \quad 1 / 100$ and filtered treatments at $28 \mathrm{~d}$.

\section{Community diversity}

245 Community diversity measured as the Shannon index showed that at $7 \mathrm{~d}$, the communities

246 from the undiluted treatment had significantly higher diversity (ANOVA, $\mathrm{p}<0.05$ ), followed 247 by the $1 / 10$ dilution, the $1 / 100$ and the filtered treatments (Fig. S1). At $14 d$, the undiluted, $248 \quad 1 / 10$ and $1 / 100$ samples had similar diversity, whereas the diversity of the filtered treatment 
community remained low. At $21 \mathrm{~d}$, the pattern of diversity did not change markedly, and at

$25028 \mathrm{~d}$, the diversity in the undiluted treatment decreased significantly.

For the phylogenetic diversity, the largest difference between samples was observed at

$2527 \mathrm{~d}$ among diluted treatments. The 1/10 communities were the more phylogenetically clustered 253 (lowest SES values), followed by the 1/100 and filtered communities (ANOVA, $\mathrm{p}<0.05$ ). As

254 time passed, communities became less phylogenetically clustered (increasing SES values), 255 and there were no significant differences between treatments (Fig. S2).

\section{Bacterial community composition inside the wood}

258 Community composition between samples was compared by computing the Bray Curtis 259 dissimilarity index. For undiluted treatment (1/1) at $7 \mathrm{~d}$, the bacterial communities were very 260 different from those of the $7 \mathrm{~d}$ communities in the diluted treatments. Dilution had a stronger 261 effect on community composition than incubation time had (Table S1 and S2). The 262 community composition changed among 7d, $14 \mathrm{~d}$ and $21 \mathrm{~d}$. However, the undiluted $21 \mathrm{~d}$ and $26328 \mathrm{~d}$ communities were very similar to each other (Fig. 2).

264 For the $1 / 10,1 / 100$ and filtered treatments, the $7 \mathrm{~d}$ communities were more similar to 265 each other than to the communities from the undiluted treatment, as shown by their close 266 proximity on the MDS plot (Fig. 2). After 7d, the 1/10 communities became more similar to 267 the 1/1 communities, whereas the $1 / 100$ and filtered communities became more similar to 268 each other with time (Fig. 2). In all dilution treatments, the communities changed with time 269 and continued changing after $21 \mathrm{~d}$. The diluted communities changed more than the undiluted 270 ones did, as shown by larger distances observed between samples on the plot. 
273 At the order level, in the diluted treatments, after 7 days (7d), there were significantly fewer

274 Campylobacterales and Flavobacteriales compared to the undiluted bacterial communities

275 (1/1). In the 1/10 dilution, the proportion of Oceanospirillales and Alteromonadales was

276 higher than that in the undiluted treatment. In the 1/100 and filtered treatments, the proportion 277 of Vibrionales was higher (Fig. 3).

278 After 14 days (14d), the samples from the undiluted treatment (1/1) maintained the

279 same groups as those present at 7 days but the proportion of Aeromonadales and

280 Alteromonadales decreased. The proportion of Campylobacterales increased significantly in

281 the 1/10 dilution. Rhodospirillales and Flavobacteriales sequences strongly increased in the $282 \quad 1 / 100$ and filtered treatments (Fig. 3).

After 21 days (21d), the proportion of Aeromonadales and Alteromonadales continued

284 to decrease in the undiluted treatment (1/1), and 2 new groups increased (Desulfovibrionales 285 and Bacteroidales). Desulfovibrionales and Bacteroidales were also detected in the 1/10

286 dilution but at lower abundance. There were no significant changes otherwise for the 1/100 287 and filtered treatments (Fig. 3).

288 After 28 days (28d), the proportion of Aeromonadales and Alteromonadales kept

289 decreasing in the undiluted treatment. In the 1/10 dilution, Desulfovibrionales and

290 Bacteroidales sequences increased. In the 1/100 dilution, Bacteroidales became more

291 abundant (Fig. 3) but more variable between replicates in the samples from the filtered 292 treatment (Fig. S3).

\section{Dynamics at the OTU level}

295 We identified OTUs representing organisms known to be involved in sulfur cycling in 296 chemosynthetic ecosystems. For sulfate reducers, we identified two OTUs that grew well in 297 the undiluted treatment but not, or poorly, in the diluted treatments (Fig. 4). These two OTUs 
were affiliated with Desulfovibrio piezophilus (OTU10 and OTU58, with 100\% and $96 \%$

299 identity, respectively, over 442 bases). OTU10 was first detected at $21 \mathrm{~d}$ in the undiluted treatment and at $28 \mathrm{~d}$ in the $1 / 10$ dilution. It was only present at trace amounts in the $1 / 100$ and

301 filtered water treatments (Fig. 4a). OTU58 was also detected in the samples at $21 \mathrm{~d}$ in the 302 undiluted treatment but was not present in the $1 / 10$ diluted treatment nor in the $1 / 100$ or 303 filtered treatments (Fig. 4b).

304 We also identified potential sulfide oxidizers OTUs that grew better in the undiluted 305 treatment than in the diluted waters (Fig. S4). These OTUs were similar to Arcobacter 306 bivalviorum (OTU6, 100\% identity) and Arcobacter sp. (OTU15, 94\% identity). OTU6 307 sequences were detected at $7 \mathrm{~d}$ in the undiluted treatment. In the $1 / 10$ dilution, sequences 308 appeared mostly at $28 \mathrm{~d}$ and were less abundant (Fig. S4a). OTU15 was only present in the 309 undiluted treatment and was detected at 21d (Fig. S4b). Some of the OTUs were more abundant in samples from the diluted compared to the 311 undiluted treatment. OTU43 (100\% identity over 452 bases to Marinomonas foliarum) was 312 more abundant at $7 \mathrm{~d}$ in the undiluted and $1 / 10$ dilution treatments but was overall more 313 abundant in the 1/100 dilution, especially from 14d (Fig. S5a). OTU19 (100\% identity over 314452 bases to Vibrio oceanisediminis) was rare in the undiluted treatment, more abundant and 315 present at all times tested in the 1/10 dilution and most abundant in the 1/100 dilution, 316 especially at 7d (Fig. S5b).

318 Proportion of sequences and cell counts

319 OTU10, OTU58, OTU6, OTU15, OTU19 and OTU43 sequences were not detected in the 320 sequences obtained from the original coastal seawater sample from Banyuls Bay. Because we 321 analyzed 23,743 sequences of bacteria from this inoculum water, the frequency of these 322 OTUs represented $<0.004 \%$ of the sequences. We further verified whether the sequences 
323

324

were present in a database of 7,418,629 sequences sampled at Banyuls Bay over a period of 9 years. OTU10, OTU58, OTU6, OTU15, OTU19 and OTU43 were never detected, and their occurrence was thus calculated to be $<0.00001 \%$.

Our flow cytometry counts validated the serial dilution of the Banyuls Bay seawater for the experiment (Fig. S6). We used the most probable number method (MPN) to estimate the number of cells belonging to the OTUs that we monitored (Table S3). Our MPN estimates showed that some OTUs had occurrences as low as 1 cell in $10 \mathrm{~L}$ of seawater (OTU15). That small proportion represents $0.00000002 \%$ of the cells present in the aquaria.

\section{OTUs in the microbial mats}

We identified OTUs affiliated with Arcobacter sp. in the mats growing on the wood logs (Fig. S7). Mats were visible starting at 21d. In the undiluted treatment, among the sequences that were identified as Arcobacter sp., the OTU Arcobacter1 dominated, followed by the OTU Arcobacter6 and a few sequences belonging to the OTU Arcobacter3 (Fig. 5). In the 1/10 dilution, the Arcobacter sequences belonged mainly to Arcobacter3. In the 1/100 dilution, very few Arcobacter sequences were present. They were represented by Arcobacter31 and Arcobacter3. In the samples from the filtered treatment, there were also few Arcobacter sequences at $21 \mathrm{~d}$ and $28 \mathrm{~d}$; half of those identified belonged to OTU1, and half belonged to OTU3. Arcobacter1 was similar to the OTU6 found inside the wood. The other 3 main Arcobacter OTUs were not detected inside the wood (Fig. 5).

\section{Discussion}

Our experiment demonstrated that seawater contains bacteria that are extremely rare but still have the potential to grow and multiply rapidly when they encounter a suitable niche. Our work suggests the existence of ultra-rare bacteria, microbes that are never detected by high 
throughput sequencing approaches but that are nevertheless viable and able to colonize new

349 ecosystems with the potential to fulfill an important function in the marine ecosystem. The presence of these ultra-rare microbes can, to date, only be revealed through

351 culturing or enrichment approaches. The organisms that grew in our experimental wood were 352 not detected by sequencing the inoculum water originating from Banyuls Bay. Their 353 occurrence in the inoculum in terms of sequence abundance was thus below $0.004 \%$. These 354 ultra-rare microbes were not found either among sequences from Banyuls Bay collected over 3558 years. By taking into account the total number of sequences from this long time series, these 356 ultra-rare microbes would represent $<0.00001 \%$ of the naturally occurring community. In terms of cell numbers, the flow cytometry counts and the most probable number approach showed that, theoretically, the frequency of ultra-rare microbes was as low as 1 cell 359 in $10 \mathrm{~L}$ of seawater or as little as $0.00000002 \%$ of the cells in the aquaria. They were not able to grow below that threshold. Together, our count and sequencing data show a level of rarity

361 that is several orders of magnitude lower than thresholds used earlier to define the rare 362 biosphere in sequencing data: 0.1\% (Pedrós-Alió 2012) or 0.01\% (Galand et al. 2009). For the 363 first time, we also provide an estimation of rarity in terms of cell numbers. Theoretically, if

364 PCR or sequencing bias were not considered, one would thus need at least $8,038,282,890$ 365 sequences (the number of bacteria present in average in $10 \mathrm{~L}$ of Banyuls Bay seawater) to be 366 able to detect the ultra-rare Arcobacter cell (Fig. 6). Thus, current sequencing techniques may 367 not provide exhaustive maps of the microbial diversity even with one million final reads, a 368 number suggested recently to be required to yield quasi-exhaustive mapping of marine 369 bacterial diversity (Crespo et al. 2016). These ultra-rare microbes could have been missed by our sequencing approach if they 371 had been present in the water as resting spores. Spore forming microbes are common (Lennon 372 \& Jones 2011) and their DNA sometimes difficult to extract. Among the sulfate-reducing 
373 bacteria, the Firmicutes form endospores that can be dispersed through the global ocean

374 (Hubert et al. 2009). However, among the bacteria that we identified, Arcobacter and

375 Desulfovibrio are considered non-spore forming bacteria (Vandamme et al. 2015) and should

376 be detected by PCR based diversity surveys. The growth of these organisms in our

377 experiments suggests that the ultra-rare biosphere contains living non-dormant bacteria, and

378 such viability shows that they do not represent taphonomic DNA originating from decaying 379 organisms, or extracellular DNA (Carini et al. 2016; Lynch \& Neufeld 2015). In the context 380 of the ongoing debate around the presence of a "inflated biosphere" originating from PCR or 381 sequencing artifacts (Kunin et al. 2010; Lee et al. 2012; Patin et al. 2013), our findings argue 382 for the true existence of the rare biosphere. In our study, we focused on microorganisms involved in the sulfur cycle. For instance, 384 sulfate reducing bacteria use the sulfate present in seawater as an electron acceptor to obtain energy and release hydrogen sulfide. By measuring the concentration of sulfide, the accumulation rates could be used to indirectly assess microbial activity and thus monitor this key ecosystem process occurring in the wood and wood surface. Our data demonstrate that 388 after diluting the inoculum 10 times, the ecosystem processes were maintained. At a 100 389 times dilution, however, sulfide was no longer detected. Thus, when ultra-rare microbes were 390 progressively removed, ecosystem processes were maintained but only until a certain 391 threshold of rarity. Below that threshold some processes were lost and ecosystem functioning 392 was impaired. Interestingly, even though sulfide was still detected after the first dilution, it 393 appeared later and the concentration plateau was not reached, with a slower buildup of sulfide 394 at the interface. It might be that at $1 / 10$ dilution, the activity started later because the 395 community had to restore a critical biomass before ecosystem processes could be detected. 396 Our data suggest, despite the fact that ecosystem functioning was maintained, that activity 397 was reduced when the original community was diluted. 
Dilution had the effect of reducing the community diversity and the phylogenetic

399 diversity at the beginning of the experiment. Dilution certainly removed some rare species

400 and probably decreased the abundance of individuals within more abundant species. It

401 affected the composition of the community used as inoculum, which in turn affected the

402 composition of the communities colonizing the wood. As a result, the composition of the

403 wood communities in the diluted treatments differed from those observed in the wood from

404 the undiluted treatment. For instance, one sulfate reducing bacteria (Desulfovibrio, OTU 58)

405 and a potential sulfide oxidizer, Arcobacter (OTU 15), were never detected in the diluted

406 treatments. Interestingly, community diversity later increased with time and the functioning of

407 the community was maintained, as revealed by the production of sulfide. It clearly shows that

408 some microorganisms from the original community were replaced by others with the potential

409 to fulfill the same metabolic tasks. Functional redundancy can be difficult to demonstrate

410 because it requires knowledge about the microbial populations that perform a specific process

411 (Allison \& Martiny 2008). Here, a change in microbial community composition did not have

412 any consequences for the microbial-mediated production of sulfide. It is an indication of the

413 functional redundancy within the bacterial species that are involved in the degradation of

414 large organic falls in the sea. Metagenomic data from planktonic marine bacteria recently

415 suggested high functional redundancy at global scale (Sunagawa et al. 2015). However, the

416 recent report that microorganisms in soils did not show a high degree of functional

417 redundancy (Fierer et al. 2013) could suggest that redundancy may vary depending on

418 ecosystems or, more likely, that the scale of the study is important. It has been emphasized

419 that redundancy has to be tested under common environmental conditions (Bradford \& Fierer 420 2012).

421 In our study, a change in community composition, concomitant with the maintenance

422 of a function, was also clearly visible in the mats growing on the wood. Our results show that 
423 at each level of dilution different Arcobacter bacterium were found in the mat. Again, it

424 points toward a certain level of functional redundancy within the genera. In addition, these

425 differences at the species level indicate that when some rare bacteria are lost or reduced in

426 abundance by dilution, other rare microbes can colonize the substrate and use the available

427 niche. Interestingly, some microorganisms that grew in the wood in the diluted treatments

428 were not detected in the woods from the undiluted treatment. For example, Marinomonas

429 (OTU43) and Vibrio oceanisediminis (OTU19) were less abundant or not detected in the

430 undiluted treatment. Dilution probably liberated a niche for these microorganisms that

431 otherwise would have been outcompeted. It should also be noted that a few Arcobacter were

432 present in the lowest dilutions after 21d, even though sulfide was not detected, which possibly

433 reflects the complete consumption of the sulfide produced and that wood could have produced

434 sulfide if the experiment had been longer.

435 In conclusion, we demonstrate the presence in the sea of very rare but viable bacteria

436 that are key to important ecosystem processes, such as organic matter degradation. These

437 functioning bacteria occurred at concentrations as low as 1 cell per $10 \mathrm{~L}$ of water and thus

438 would not be detected by current amplicon based environmental diversity surveys. In the

439 debate on the extent of microbial diversity, we found that the bacterial community richness

440 remains under estimated (Crespo et al. 2016; Locey \& Lennon 2016). The rare biosphere

441 described to date only represents the emerged part of an iceberg floating in a sea of ultra-rare

442 microbes, which represent an underappreciated source of hidden diversity.

444 Acknowledgment. The work of PEG was supported by the Agence Nationale de la

445 Recherche (ANR) through the projects EUREKA (ANR-14-CE02-0004-01). We

446 acknowledge the support of Fondation Total to the UPMC 'chair biodiversity, extreme marine 
environment and global change'. We are thankful to Connie Lovejoy for helpful advices and

448 comments on the manuscript.

Author contributions. P.E.G. and D.K. designed research, D.K, E.P., N.L.B. and P.E.G.

performed research, P.E.G contributed new reagents/analytic tools, D.K, N.L.B. and P.E.G. analyzed data, D.K, E.P., N.L.B. and P.E.G. wrote the paper.

453

Data Accessibility. The raw sequence data have been deposited in the NCBI Sequence Read

Archive (accession no. SRP099419 and BioProject accession no. PRJNA374511).

\section{References}

Allison SD, Martiny JBH (2008) Resistance, resilience, and redundancy in microbial communities. Proceedings of the National Academy of Sciences, 105, 11512-11519.

Alonso - Sáez L, Díaz - Pérez L, Morán XAG (2015) The hidden seasonality of the rare biosphere in coastal marine bacterioplankton. Environmental Microbiology, 17, 3766-3780.

Amann R, Rossello-Mora R (2016) Reply to "The Underestimation of Global Microbial Diversity". mBio, 7, e01623-01616.

Amann R, Rosselló-Móra R (2016) After all, only millions? mBio, 7, e00999-00916.

Bell T, Newman JA, Silverman BW, Turner SL, Lilley AK (2005) The contribution of species richness and composition to bacterial services. Nature, 436, 1157-1160.

Bienhold C, Pop Ristova P, Wenzhöfer F, Dittmar T, Boetius A (2013) How Deep-Sea Wood Falls Sustain Chemosynthetic Life. PLoS ONE, 8, e53590.

Bradford MA, Fierer N (2012) The biogeography of microbial communities and ecosystem processes: implications for soil and ecosystem models. In: Soil Ecology and Ecosystem Services (eds. Wall DH, Bardgett RD), p. 424. Oxford University Press, Oxford.

Campbell BJ, Engel AS, Porter ML, Takai K (2006) The versatile \&epsi;-proteobacteria: key players in sulphidic habitats. Nature Reviews Microbiology, 4, 458-468.

Campbell BJ, Yu L, Heidelberg JF, Kirchman DL (2011) Activity of abundant and rare bacteria in a coastal ocean. Proceedings of the National Academy of Sciences, 108, 12776-12781.

Carini P, Marsden PJ, Leff JW, et al. (2016) Relic DNA is abundant in soil and obscures estimates of soil microbial diversity. bioRxiv, 043372.

Crespo BG, Wallhead PJ, Logares R, Pedrós-Alió C (2016) Probing the Rare Biosphere of the North-West Mediterranean Sea: An Experiment with High Sequencing Effort. PLOS ONE, 11, e0159195. 
Curtis TP, Sloan WT, Scannell JW (2002) Estimating prokaryotic diversity and its limits. Proceedings of the National Academy of Sciences, 99, 10494-10499.

Edgar RC (2004) MUSCLE: a multiple sequence alignment method with reduced time and space complexity. BMC Bioinformatics, 5, 113.

Edgar RC (2010) Search and clustering orders of magnitude faster than BLAST. Bioinformatics, 26, 2460-2461.

Falkowski PG, Fenchel T, Delong EF (2008) The microbial engines that drive Earth's biogeochemical cycles. Science, 320, 1034-1039.

Fierer N, Ladau J, Clemente JC, et al. (2013) Reconstructing the microbial diversity and function of pre-agricultural tallgrass prairie soils in the United States. Science, 342, 621-624.

Finlay BJ, Maberly SC, Cooper JI (1997) Microbial diversity and ecosystem function. Oikos, 209-213.

Galand P, Salter I, Kalenitchenko D (2015) Microbial productivity is associated with phylogenetic distance in surface marine waters Molecular Ecology, 24, 57855795.

Galand PE, Casamayor EO, Kirchman DL, Lovejoy C (2009) Ecology of the rare microbial biosphere of the Arctic Ocean. Proceedings of the National Academy of Sciences of the United States of America, 106, 22427-22432.

Garcia SL, Buck M, McMahon KD, et al. (2015) Auxotrophy and intrapopulation complementary in the 'interactome' of a cultivated freshwater model community. Molecular Ecology, 24, 4449-4459.

Giovannoni SJ, Thrash JC, Temperton B (2014) Implications of streamlining theory for microbial ecology. The ISME journal, 8, 1553-1565.

Hammer Ø, Harper DAT, Ryan PD (2001) PAST: Paleontological statistics software package for education and data analysis. Palaeontologia Electronica, 4, 9.

Hubert C, Loy A, Nickel M, et al. (2009) A constant flux of diverse thermophilic bacteria into the cold Arctic seabed. Science, 325, 1541-1544.

Hugoni M, Taib N, Debroas D, et al. (2013) Structure of the rare archaeal biosphere and seasonal dynamics of active ecotypes in surface coastal waters. Proceedings of the National Academy of Sciences of the United States of America, 110, 6004-6009.

Jones SE, Lennon JT (2010) Dormancy contributes to the maintenance of microbial diversity. Proceedings of the National Academy of Sciences, 107, 5881-5886.

Jørgensen BB (1982) Mineralization of organic matter in the sea bed-the role of sulphate reduction. Nature, 296, 643-645.

Kalenitchenko D, Dupraz M, Le Bris N, et al. (2016) Ecological succession leads to chemosynthesis in mats colonizing wood in sea water. The ISME journal, 10, 2246-2258.

Kalenitchenko D, Fagervold SK, Pruski AM, et al. (2015) Temporal and spatial constraints on community assembly during microbial colonization of wood in seawater. The ISME journal, 9, 2657-2670.

Kalenitchenko D, Le Bris N, Dadaglio L, et al. (2017) Bacteria alone establish the chemical basis of the wood-fall chemosynthetic ecosystem in the deep-sea. The ISME journal, in press.

Kembel SW (2009) Disentangling niche and neutral influences on community assembly: assessing the performance of community phylogenetic structure tests. Ecology Letters, 12, 949-960.

Kembel SW, Cowan PD, Helmus MR, et al. (2010) Picante: R tools for integrating phylogenies and ecology. Bioinformatics, 26, 1463-1464. 
Kirchman DL (2010) Microbial ecology of the oceans John Wiley \& Sons.

Kunin V, Engelbrektson A, Ochman H, Hugenholtz P (2010) Wrinkles in the rare biosphere: pyrosequencing errors can lead to artificial inflation of diversity estimates. Environmental Microbiology, 12, 118-123.

Laurent MC, Gros O, Brulport J-P, Gaill F, Le Bris N (2009) Sunken wood habitat for thiotrophic symbiosis in mangrove swamps. Marine Environmental Research, 67, 83-88.

Le Bris N, Contreira-Pereira L, Yücel M (2012) In situ sensors for benthic ecosystem studies. In: Sensors for Ecology: Towards Integrated Knowledge of Ecosystems (eds. Le Galliard JF, Guarini JM, Gaill F), pp. 185-208. CNRS, Paris.

Lee CK, Herbold CW, Polson SW, et al. (2012) Groundtruthing next-gen sequencing for microbial ecology-biases and errors in community structure estimates from PCR amplicon pyrosequencing. PLOS ONE, 7, e44224.

Leff JW, Jones SE, Prober SM, et al. (2015) Consistent responses of soil microbial communities to elevated nutrient inputs in grasslands across the globe. Proceedings of the National Academy of Sciences, 112, 10967-10972.

Lennon JT, Jones SE (2011) Microbial seed banks: the ecological and evolutionary implications of dormancy. Nature Reviews Microbiology, 9, 119-130.

Lennon JT, Locey KJ (2016) The underestimation of global microbial diversity. mBio, 7, e01298-01216.

Locey KJ, Lennon JT (2016) Scaling laws predict global microbial diversity. Proceedings of the National Academy of Sciences, 113, 5970-5975.

Lynch MD, Neufeld JD (2015) Ecology and exploration of the rare biosphere. Nature Reviews Microbiology, 13, 217-229.

Martin W, Baross J, Kelley D, Russell MJ (2008) Hydrothermal vents and the origin of life. Nature Reviews Microbiology, 6, 805-814.

McDonald JH (2014) Handbook of Biological Statistics Sparky House Publishing, Baltimore.

McFall-Ngai M, Hadfield MG, Bosch TC, et al. (2013) Animals in a bacterial world, a new imperative for the life sciences. Proceedings of the National Academy of Sciences, 110, 3229-3236.

Morris JJ, Lenski RE, Zinser ER (2012) The Black Queen Hypothesis: evolution of dependencies through adaptive gene loss. mBio, 3, e00036-00012.

Oblinger J, Koburger J (1975) Understanding and teaching the most probable number technique. Journal of Milk and Food Technology (JMFT), 38, 540-545.

Patin NV, Kunin V, Lidström U, Ashby MN (2013) Effects of OTU clustering and PCR artifacts on microbial diversity estimates. Microbial Ecology, 65, 709-719.

Pedrós-Alió C (2006) Marine microbial diversity: can it be determined? Trends in Microbiology, 14, 257.

Pedrós-Alió C (2012) The rare bacterial biosphere. Annual Review of Marine Science, 4, 449-466.

Price MN, Dehal PS, Arkin AP (2010) FastTree 2-approximately maximum-likelihood trees for large alignments. PLoS ONE, 5, e9490.

Pruesse E, Quast C, Knittel K, et al. (2007) SILVA: a comprehensive online resource for quality checked and aligned ribosomal RNA sequence data compatible with ARB. Nucleic Acids Research, 35, 7188-7196.

Rickard D, Luther GW (2007) Chemistry of iron sulfides. Chemical reviews, 107, 514-562. 
Sogin ML, Morrison HG, Huber JA, et al. (2006) Microbial diversity in the deep sea and the under explored "rare biosphere". Proceedings of the National Academy of Sciences of the United States of America, 103, 12115-12120.

Sunagawa S, Coelho LP, Chaffron S, et al. (2015) Structure and function of the global ocean microbiome. Science, 348, 1261359.

Vandamme P, Dewhirst FE, Paster BJ, On SLW (2015) Arcobacter. In: Bergey's Manual of Systematics of Archaea and Bacteria. John Wiley \& Sons, Ltd.

Webb CO, Ackerly DD, Kembel SW (2008) Phylocom: software for the analysis of phylogenetic community structure and trait evolution. Bioinformatics, 24, 20982100.

\section{Figure legends}

Figure 1. (a) Electrode potential as function of time indicating the presence of sulfide on the surface of wood inoculated with different dilutions of seawater $(1 / 1,1 / 10,1 / 100$ and filtered).

A decrease in electrode potential indicates an accumulation of sulfide. $7 \mathrm{~d}, 14 \mathrm{~d}, 21 \mathrm{~d}$ and $28 \mathrm{~d}$ indicate sampling times. (b) Microprofiles of sulfide concentration through the mats colonizing the wood surface in the undiluted (1/1) (black lines) and 1/10 dilution experiments (grey lines) measured after 21 days (dashed lines) and 28 days (full lines). Error bars represent the standard deviation obtained from triplicate measurements.

Figure 2. Trajectories of the bacterial communities. The similarity between bacterial communities growing inside the wood is represented by an MDS ordination based on the Bray-Curtis index. Colors group communities sampled from a same dilution treatment. The size of the points is proportional to the time elapsed after the incubation started. The percent value for each axis represents the proportion of total variation explained.

Figure 3. Proportions of sequences at the order level detected inside woods incubated in aquaria inoculated with seawater with a range of dilutions $(1 / 1,1 / 10,1 / 100$ and only filtered) and sampled at four different time points (7 to 28 days). Each bar shows the average values of 4 replicate samples. 
611 Figure 4. Abundance of sequences belonging to two sulfate reducing bacteria identified as

612 Desulfovibrio piezophilus OTU10 (a) and Desulfovibrio sp. OTU58 (b) during the experiment

613 in the 4 different inoculation conditions. The number of sequences represents averages over

614 four replicates.

615

616 Figure 5. Proportion of the 4 most abundant Arcobacter OTUs in the mats colonizing the

617 wood surface in the 4 different treatment conditions $(1 / 1,1 / 10,1 / 100$ and filtered) at $21 \mathrm{~d}$ and

618 28d. The proportion is relative to the total number of sequences found in each sample. The

619 whole wood surface was sampled for sequencing.

620

621

622

623

624

625

626

627

628

629

630

631

632

633

634 


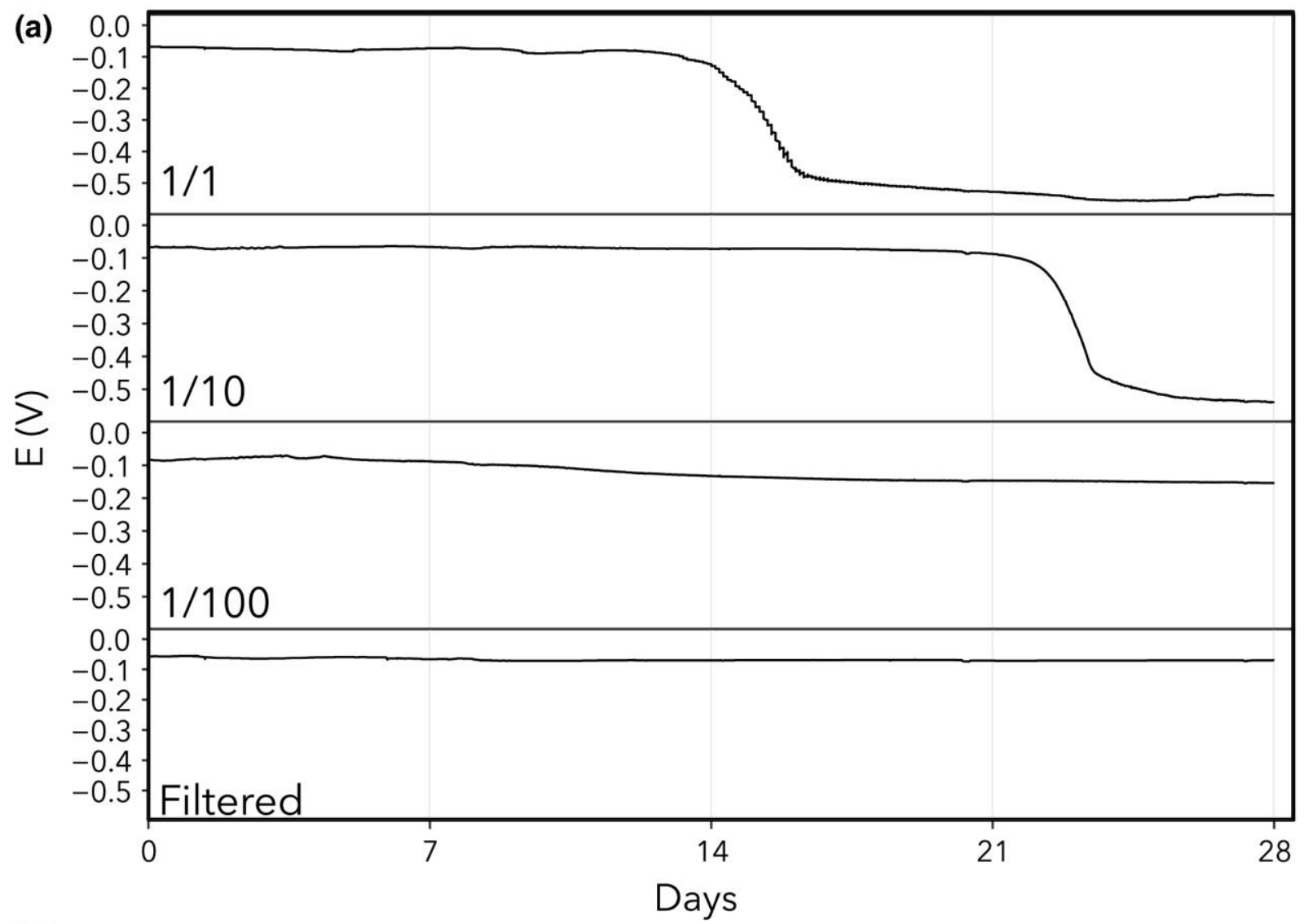

(b)

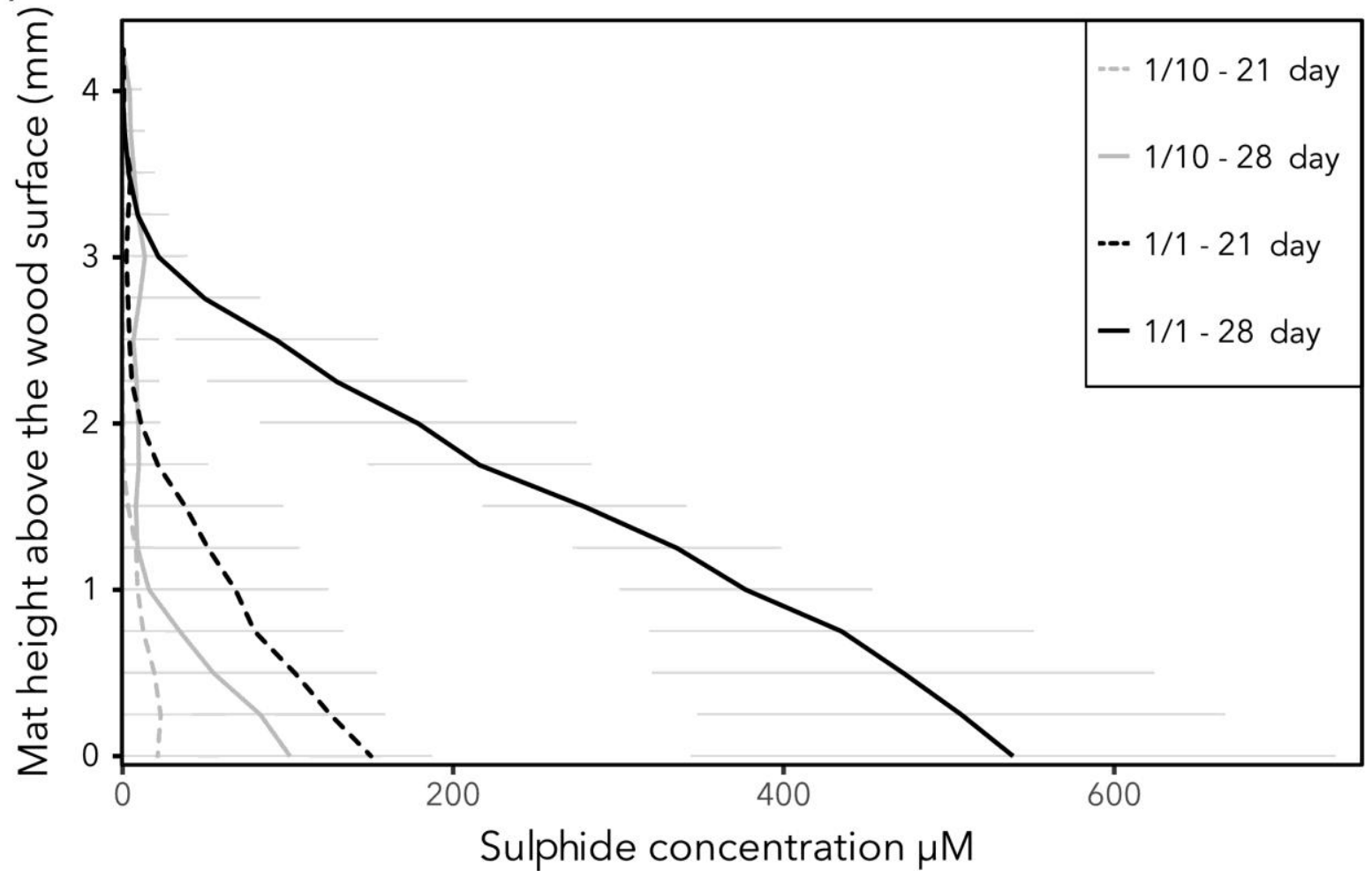

Fig.1 
643

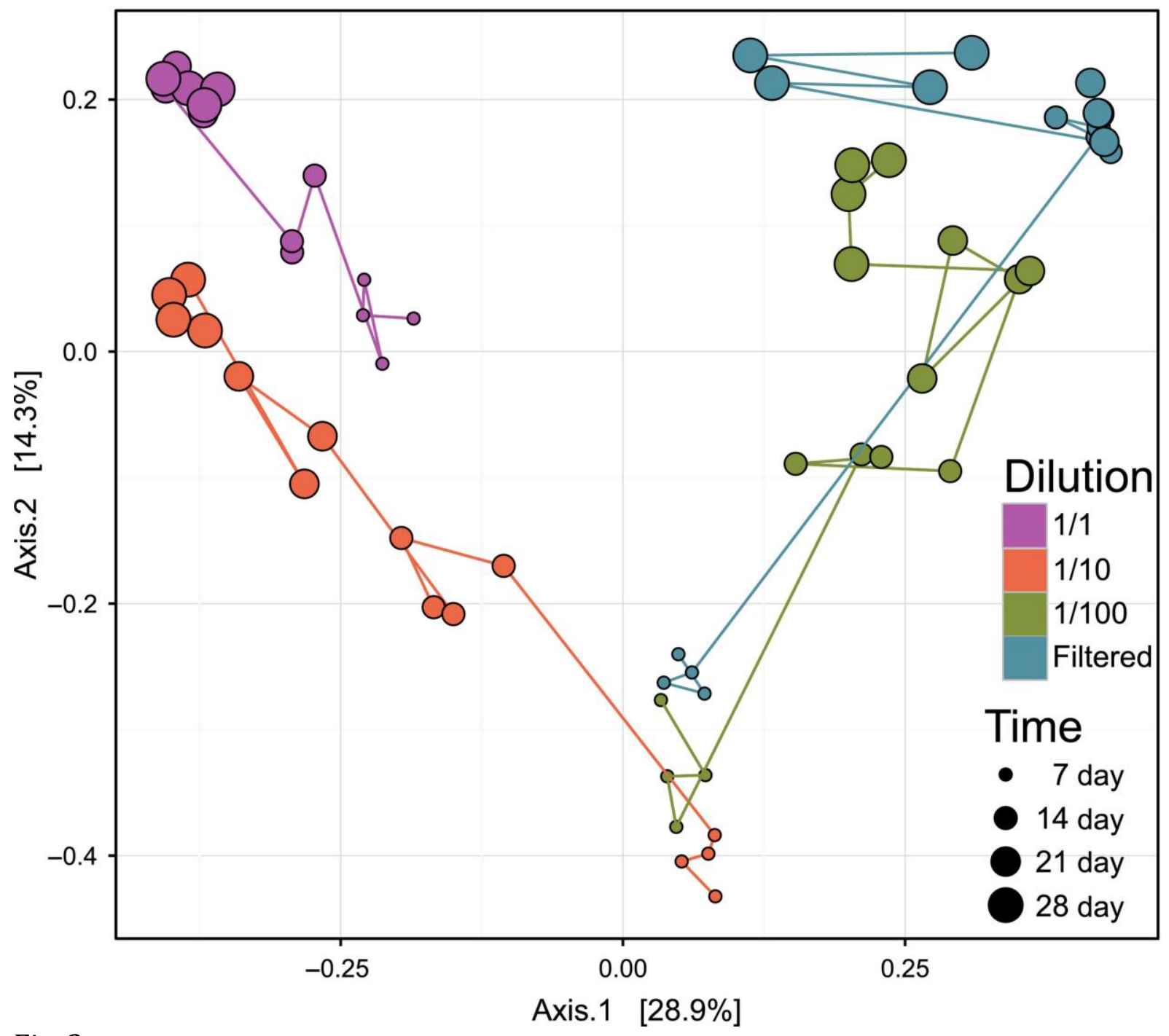

Fig. 2 
647 Fig. 3

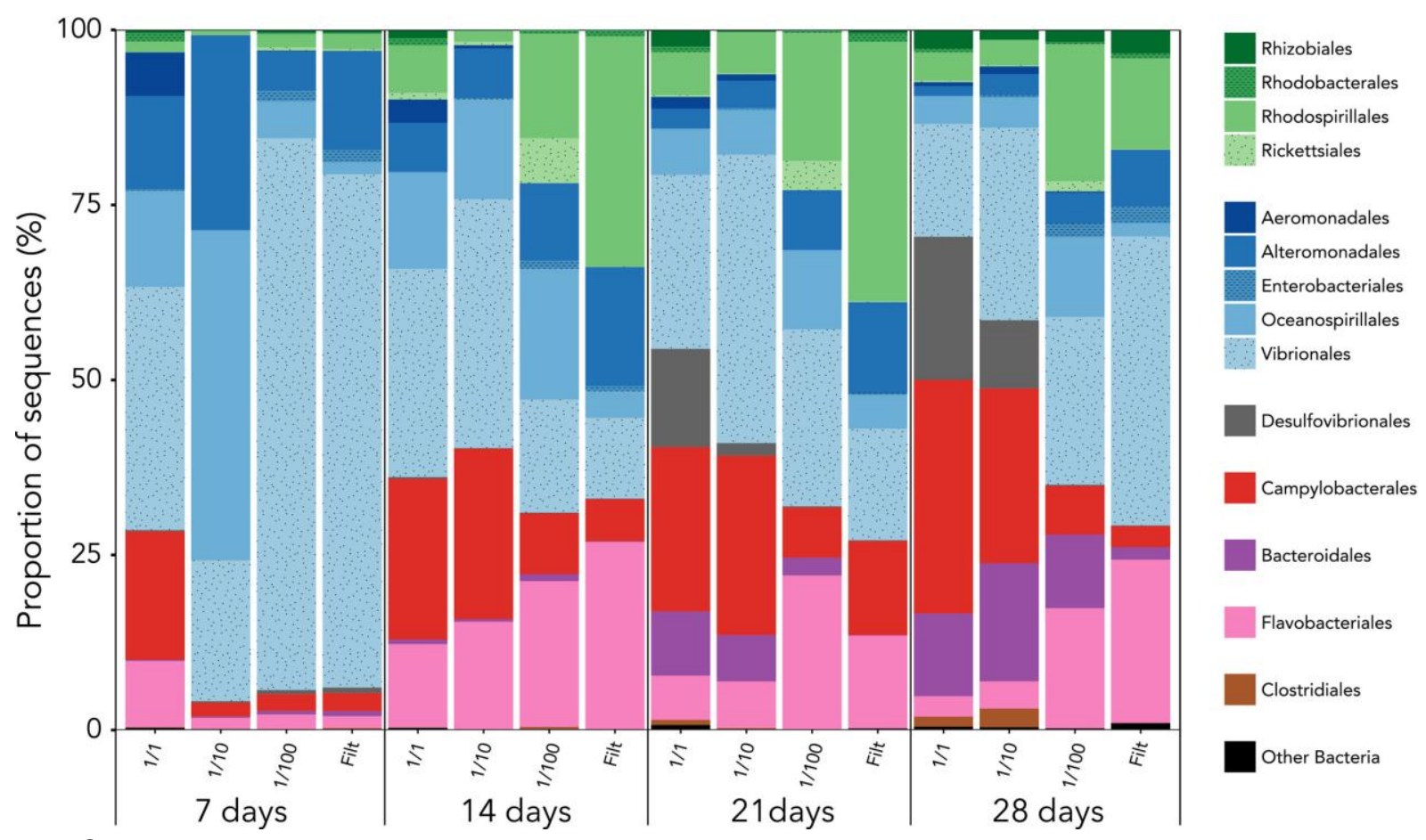

648

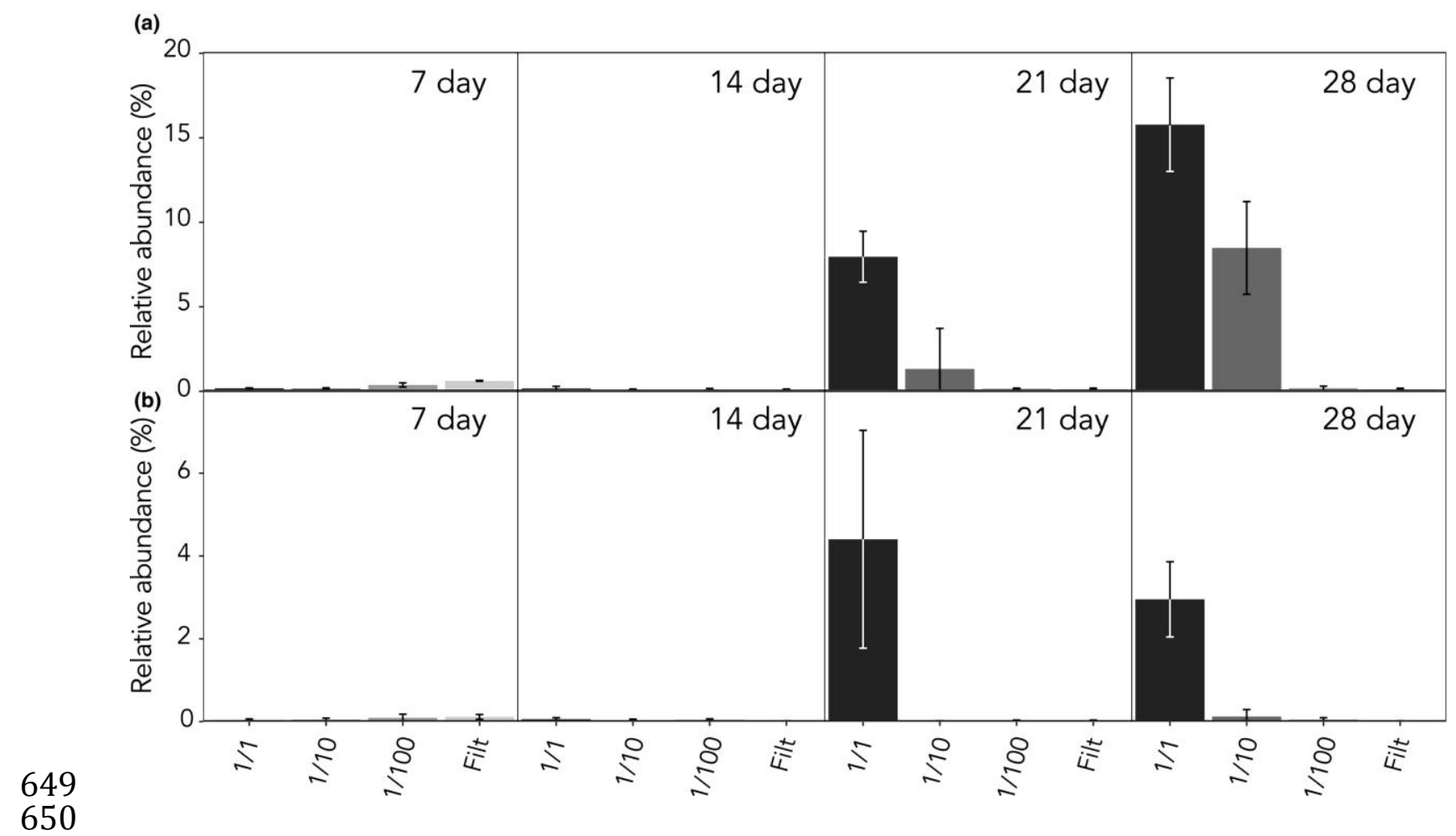

651 Fig. 4

652 


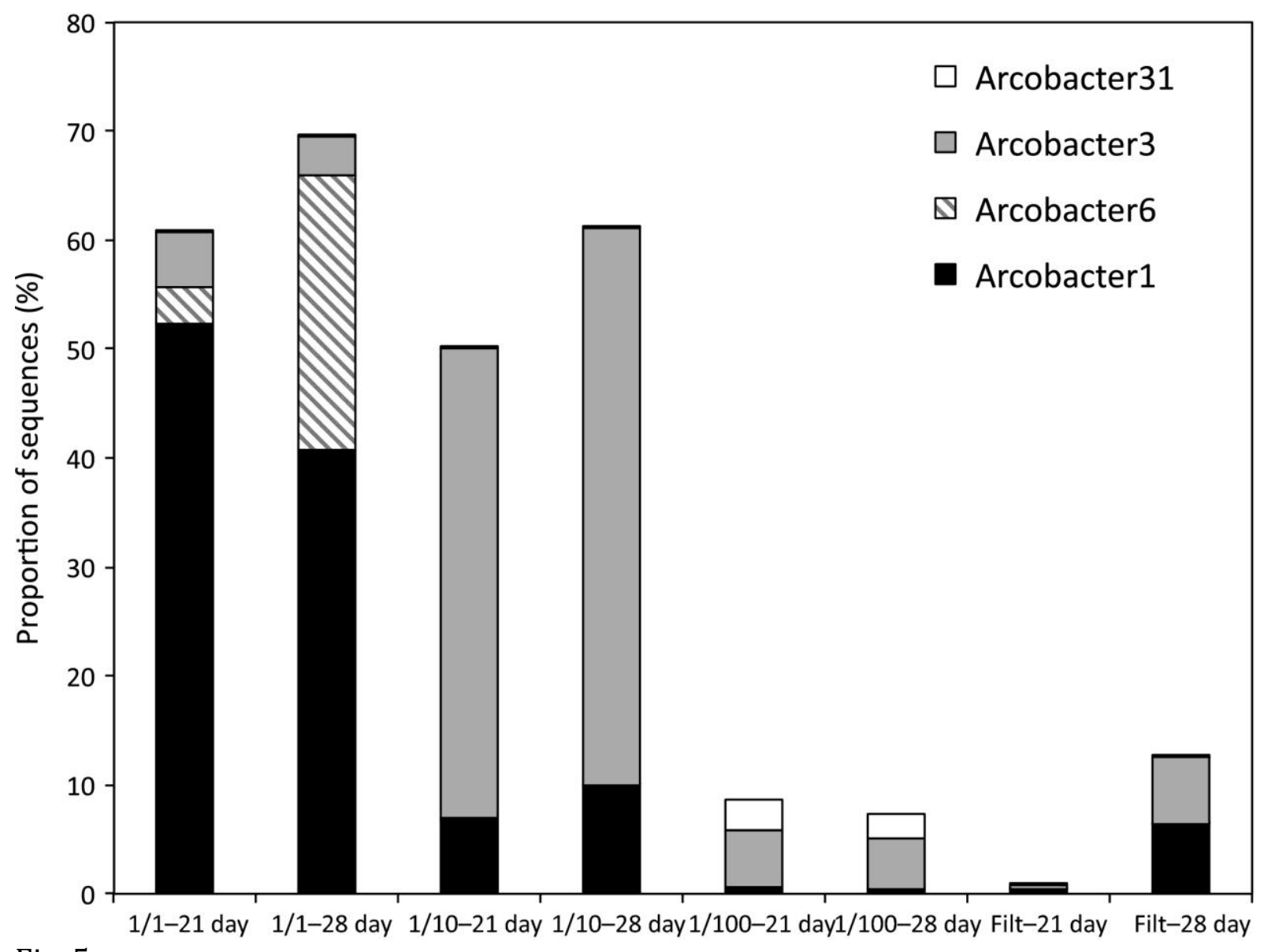

653 654 Fig. 5

655

656

657

658

659

660

661

662

663 